\title{
Modified Pulsatilla decoction attenuates oxazolone-induced colitis in mice through suppression of inflammation and epithelial barrier disruption
}

\author{
XUEWEI WANG, FUGANG FAN and QIN CAO \\ Department of Gastroenterology, Putuo Hospital, Shanghai University of Traditional Chinese Medicine, \\ Shanghai 200062, P.R. China \\ Received July 1, 2015; Accepted May 5, 2016
}

DOI: $10.3892 / \mathrm{mmr} .2016 .5358$

\begin{abstract}
Inflammatory bowel diseases (IBDs) are chronic inflammatory gastrointestinal disorders caused by a dysregulated mucosal immune response and epithelial barrier disruption. Conventional treatment of IBD is currently limited to overcoming patient symptoms and is often associated with severe adverse effects from the drugs used. Modified Pulsatilla decoction has been used previously to treat ulcerative colitis (UC) in clinical practice in China, however, the underlying mechanism in the treatment of UC remains to be elucidated. In the present study, the efficiency and mechanisms of modified Pulsatilla decoction in the treatment of oxazolone-induced colitis were investigated. Assessment of clinical colitis and histological examination found that the administration of modified Pulsatilla decoction attenuated the severity of oxazolone-induced colitis in mice. Measurement of cytokine concentration, western blotting and reverse transcription-quantitative polymerase chain reaction demonstrated modified Pulsatilla decoction treatment significantly reduced the secretion of pro-inflammatory cytokines and restored alterations in tight junction proteins in the colon tissues. In addition, modified Pulsatilla decoction suppressed the activation of the nuclear factor- $\kappa \mathrm{B}$ signaling pathway. Thus, the findings of the present study demonstrated that modified Pulsatilla decoction offers an effective therapeutic approach for the treatment of IBD and revealed the underlying mechanisms of action offered by modified Pulsatilla decoction.
\end{abstract}

Correspondence to: Professor Qin Cao, Department of Gastroenterology, Putuo Hospital, Shanghai University of Traditional Chinese Medicine, 164 Lanxi Road, Shanghai 200062, P.R. China

E-mail: qincao201506@163.com

Key words: pulsatilla decoction, oxazolone, colitis, inflammation

\section{Introduction}

Inflammatory bowel diseases (IBDs) are idiopathic inflammatory gastrointestinal (GI) disorders, which consist of Crohn's disease (CD) and ulcerative colitis (UC). It is currently accepted that alterations in the gut microbiota, genetic susceptibility, dysfunction of immunological regulation and defects in the intestinal barrier are involved in the etiopathogenesis of IBD $(1,2)$. In general, UC is solely restricted to the colon, whereas CD may affect the entire GI tract. Abdominal pain, weight loss and bloody diarrhea are the most common symptoms of IBD (3). IBDs are lifelong diseases, for which patients often require lifelong pharmacological treatment and may require surgical interventions.

The conventional medications used for the treatment of IBD include corticosteroids, immunosuppressive drugs, antibiotics and biological agents, including anti-tumor necrosis factor- $\alpha$. However, the use of these drugs is only effective for temporary symptomatic relief, and is associated with serious complications and toxic side effects (3-5). Thus, the use of complementary and alternative medicine (CAM), including acupuncture, homeopathy and herbal medicines, for the treatment of patients with IBD is increasing worldwide, and the percentage of patients with IBD using CAM in North America and Europe has been estimated at up to 70\% (6-9). Traditional Chinese medicine (TCM) is one of the most developed branches of CAM in the world (10). Several scientific reports and clinical studies have characterized the effectiveness of herbal preparations in IBD, showing promising results and providing an insight into the mechanisms, which are beyond their therapeutic activity (11-14).

Modified Pulsatilla decoction, containing seven commonly used plants (Radix Pulsatillae, Cortex Phellodendri, Rhizoma Coptidis, Cortex Fraxini, Sanchi, Radix Paeoniae Rubra and Radix Glycyrrhizae), is formulated using the TCM formulation of Pulsatilla decoction, and adding another three herbal plants (Sanchi, Radix Paeoniae Rubra and Radix Glycyrrhizae). Pulsatilla decoction possesses a variety of pharmacological effects, and the active ingredients from these herbal plants have shown hepatic protective, anti-inflammatory, antibacterial, antitumor and anti-oxidant effects (15-19). Modified Pulsatilla decoction has been used previously to treat UC in 
clinical practice in China, showing promising effects against inflammation and diarrhea (20). Although modified Pulsatilla decoction is an effective TCM formula for the pharmacological effects indicated above, the possible mechanism in the treatment of UC remains to be elucidated. Thus, the present study aimed to investigate the therapeutic efficacy of modified Pulsatilla decoction against IBD and to elucidate the underlying molecular mechanisms.

\section{Materials and methods}

Induction of colitis. A total of 40 male BALB/c mice (age, 6 weeks; weight, 21-24 g) were obtained from Shanghai Laboratory Animal Center (Shanghai, China) and housed in a specific-pathogen-free environment under normal conditions of humidity $(50 \pm 5 \%)$ and temperature $\left(25 \pm 2^{\circ} \mathrm{C}\right)$, with a 12 -h light/dark cycle and access to food and water ad libitum. The mice were randomly divided into 4 groups of 10 , the control, PBS, modified Pulsatilla and sulfasalazine (SASP; Pfizer, Inc., New York, NY, USA). Colitis was induced, as previously described, with modifications (21). Briefly, a $2 \times 2 \mathrm{~cm}$ field of the abdominal skin was shaved, and $200 \mu 13 \%$ oxazolone (Sigma-Aldrich, St. Louis, MO, USA) in $100 \%$ ethanol was applied to pre-sensitize the BALB/c mice, and this was repeated the following day. Subsequently, a solution of oxazolone $(150 \mu \mathrm{l}$; $1 \%$ oxazolone dissolved in $50 \%$ ethanol) was administered into the colon via the anus using a $3.5-\mathrm{F}$ polyurethane catheter. Following injection of the oxazolone solution, the catheter was removed and the mouse was held vertically for $60 \mathrm{sec}$. In the control group, the same volume of ethanol was injected, instead of the oxazolone solution. Following treatment, all mice were fed a standard chow diet and tap water ad libitum. The present study was approved by the ethics committee of Shanghai University of Traditional Chinese Medicine (Shanghai, China) and performed in accordance with the Guide for the Care and Use of Laboratory Animals (22).

Preparation and intragastric administration of water-soluble extracts of modified Pulsatilla decoction. The modified Pulsatilla decoction consisted of Radix Pulsatillae (30 g), Cortex Phellodendri (12 g), Rhizoma Coptidis (5 g), Cortex Fraxini (9 g), Sanchi (3 g), Radix Paeoniae Rubra (12 g) and Radix Glycyrrhizae (6 g). To obtain the water-soluble extracts, 10 -fold higher volumes of water was added to these herbal plants, and the resulting mixtures were then heated at $90^{\circ} \mathrm{C}$ for $1 \mathrm{~h}$. This procedure was repeated three times, following which all the extraction liquids were collected, dried and stored at $4^{\circ} \mathrm{C}$.

The extracts of the decoction $(10 \mathrm{mg} / \mathrm{g}$ body weight $)$ or SASP (20 mg/g body weight) were administered intragastrically to the oxazolone-induced colitis mice once a day for 7 days. SASP acted as a positive control. As a negative control, an equal volume of water was administered to the oxazolone-induced colitis mice. The PBS group were oxazolone-induced colitis mice administered $\sim 1 \mathrm{ml}$ PBS.

Assessment of clinical colitis in mice. The seventy of colitis was assessed using a disease activity index (DAI) scoring system and loss in body weight. The DAI score was calculated as the sum of the bloody stool score $(0$, normal colored stool;
1, brown stool; 2. reddish stool; 3 , bloody stool) and diarrheal stool score ( 0 , normal stool; 1 , mildly soft stool; 2 , very soft stool; 3 , watery stool). The body weight change in each mouse was calculated using the following formula: Weight loss $(\%)=[($ weight on day 1$)-($ weight each day $)] /$ weight on day 1 .

Histological examination of colon. After 7 days, the mice were sacrificed using $0.1 \mathrm{ml} 2 \%$ sodium pentobarbital and a $10 \mathrm{~cm}$ portion of the distal colon was removed from 4 mice in each group, washed with saline and fixed in $10 \%$ natural buffered formalin. The fixed tissues were then embedded in paraffin (Sigma-Aldrich), cut into tissue sections (5 $\mu \mathrm{m}$ thick) and stained with hematoxylin and eosin (Leica Microsystems, Ltd., Milton Keynes, UK) and examined with a microscope (Leica Microsystems, Ltd.) and qualitatively evaluated with Leica QWin software 3.1 using the following parameters: Transmural infiltrating of mononuclear cells, thickening of the colon wall, mucosal hyperplasia, erosions, depletion of goblet cells and crypt architectural distortion.

Reverse transcription-quantitative polymerase chain reaction $(R T-q P C R)$ analysis. Frozen colon samples were homogenized in $1 \mathrm{ml}$ cold buffer containing $0.1 \mathrm{M}$ phosphate buffer (pH 7.4) and $1 \%$ protease inhibitor cocktail (Sigma-Aldrich). The homogenized samples were centrifuged at $12,000 \mathrm{x} \mathrm{g}$ for $15 \mathrm{~min}$ at $4^{\circ} \mathrm{C}$ and the supernatants were collected and stored at $-80^{\circ} \mathrm{C}$. Total RNA was isolated from the colon tissues using TRIzol reagent (Invitrogen; Thermo Fisher Scientific, Inc., Waltham, MA, USA) and $1.5 \mu \mathrm{g}$ RNA was reverse transcribed into cDNA using a GoScript ${ }^{\mathrm{TM}}$ Reverse Transcription system (Promega Corporation, Madison, WI, USA), according to the manufacturer's protocol. The RT-qPCR analysis was performed to determine the expression levels of interleukin (IL)-5 and IL-13 by using SYBR green PCR master mix (Applied Biosystems; Thermo Fisher Scientific, Inc.). The primers for IL-5 were: Sense 5'-AAGGATGCTTCTGCACTTGA-3' and antisense 5'-GGAAGCCTCATCGTCTCATT-3'; the primers for IL-13 were: Sense 5'-AGCATGGTATGGAGTGTGGA-3' and antisense 5'-TTGCAATTGGAGATGTTGGT-3'; and the primers for GAPDH were: Sense 5'-AGGTCGGTGTGAACG GATTTG-3' and antisense 5'-TGTAGACCATGTAGTTGA GGTCA-3'. The PCR reaction was performed on an iCycler Real-Time PCR Detection system (Bio-Rad Laboratories, Inc., Hercules, CA, USA) with thermocycling conditions as follows: $95^{\circ} \mathrm{C}$ for $5 \mathrm{~min}$; 40 cycles of $95^{\circ} \mathrm{C}$ for $15 \mathrm{sec}, 60^{\circ} \mathrm{C}$ for $15 \mathrm{sec}$ and $72^{\circ} \mathrm{C}$ for $20 \mathrm{sec}$. The fluorescence intensity was determined by the CFX96 Real-Time PCR Detection system (Bio-Rad Laboratories, Inc.) The relative expression ratio of cytokines was calculated using the $2^{-\Delta \Delta \mathrm{Cq}}$ method (23).

ELISA. The protein levels of IL-5 and IL-13 in the supernatants of the colon tissue lysates were measured using Mouse IL-13 Quantikine ELISA kit and Mouse IL-5 Quantikine ELISA kit (R\&D Systems, Minneapolis, MN, USA), according to the manufacturer's protocol. All samples were analyzed in duplicate and normalized to the total protein content of the sample, expressed as $\mathrm{pg} / \mathrm{mg}$ protein.

Western blot analysis. Frozen colonic tissues were mechanically homogenized $(20 \% \mathrm{w} / \mathrm{v})$ in lysis buffer (pH 7.4) containing 
$0.1 \mathrm{mM}$ EDTA, $20 \mathrm{mM}$ HEPES, $12.5 \mathrm{mM} \mathrm{MgCl} \mathrm{m}_{2}, 150 \mathrm{mM}$ $0.1 \%$ Nonidet $\mathrm{P} 40, \mathrm{NaCl}, 1 \mathrm{mM}$ dithiothreitol, $0.2 \mathrm{mM}$ phenylmethylsulfonyl fluoride, and $1 \mu \mathrm{g} / \mathrm{ml}$ leupeptin, aprotinin, and pepstatin. Homogenates were sonicated and then centrifuged for $10 \mathrm{~min}$ and $12,000 \mathrm{x}$ g. Protein concentration was assessed using the Bradford protein assay. Equivalent quantities $(20 \mu \mathrm{g})$ of protein from the cells lysates were loaded onto SDS-PAGE gels (Beyotime Institute of Biotechnology, Haimen, China) and transferred onto polyvinylidene fluoride membranes (EMD Millipore, Billerica, MA, USA). The membranes were blocked with $5 \%$ non-fat milk in Tris-buffered saline, and then incubated with primary antibodies overnight at $4^{\circ} \mathrm{C}$, followed by incubation at room temperature for $2 \mathrm{~h}$ with goat anti-rabbit horseradish peroxidase-conjugated secondary antibody (Beyotime Institute of Biotechnology; cat. no. A0208; dilution, 1:5,000). Signals were detected using enhanced chemiluminesecence reagent (EMD Millipore), rabbit polyclonal ZO-1 (cat. no. 40-2200; dilution, 1:1,000), rabbit polyclonal claudin-2 (cat. no. PA5-13335; dilution, 1:1,000) and rabbit monoclonal occludin antibodies (cat. no. 710192; dilution, 1:1,000) were purchased from Invitrogen (Thermo Fisher Scientific, Inc.), rabbit monoclonal inhibitor of NF- $\kappa \mathrm{B}-\alpha(\mathrm{I} \kappa \mathrm{B}-\alpha)$ antibody was purchased from Cell Signaling Technology, Inc. (Danvers, MA, USA; cat. no. 4812; dilution, 1:1,000) and the rabbit polyclonal GAPDH antibody was purchased from Santa Cruz Biotechnology, Inc. (Santa Cruz, CA, USA; cat. no. sc-25778; dilution, 1:1,000).

Data analysis. Results are presented as the mean \pm standard deviation. All data presented are representative of three or more experiments, each with similar results. Statistical analysis was conducted using SPSS 19.0 (IBM SPSS, Armonk, NY, USA) and significance was determined using Student's test. $\mathrm{P}<0.05$ was considered to indicate a statistically significant difference.

\section{Results}

Modified Pulsatilla decoction treatment attenuates the severity of oxazolone-induced colitis in mice. To investigate the therapeutic effect of modified Pulsatilla decoction on IBD, the present study induced experimental colitis in mice via skin pre-sensitization and colonic administration of oxazolone. The results demonstrated that the mice injected with oxazolone developed experimental colitis with severe weight loss (Fig. 1A), severe hunching, lethargy, piloerection and diarrhea, and showed a significantly increased DAI score, compared with the normal group $(\mathrm{P}<0.01)$. Although there were no differences between the Pulsatilla decoction-treated mice and the SASP-treated mice, oral administration of modified Pulsatilla decoction and SASP significantly attenuated the severity of oxazolone-induced colitis, which showed decreased DAI scores, compared with the untreated group $(\mathrm{P}<0.01$; Fig. 1B).

The severity of the histological changes in the colon were then qualitatively evaluated in the mice treated with or without modified Pulsatilla decoction or SASP. As shown in Fig. 1C, the colonic histological changes were characterized by transmural infiltrating of mononuclear cells, thickening of the colon wall, mucosal hyperplasia, erosions, depletion of goblet cells and crypt architectural distortion. Compared with the modified Pulsatilla decoction-treated mice and SASP-treated mice, the severity of damage was more marked in the untreated mice. However, the colonic tissues in the Pulsatilla decoction-treated and SASP-treated mice showed marked improvements in inflammation. Together, these findings indicated that the modified Pulsatilla decoction treatment attenuated the severity of oxazolone-induced colitis in the mice.

Modified Pulsatilla decoction treatment significantly reduces the secretion of pro-inflammatory cytokines. As the excessive production of pro-inflammatory cytokines is closely correlated with tissue damage and is critical in the pathogenesis of IBD (24), the present study characterized the expression levels of inflammatory cytokines in the colonic mucosal tissues. As shown in Fig. 2A and B, the expression levels of IL-5 and IL-13 were low in the colon tissues of the normal mice, and markedly upregulated in the colon tissues of the oxazolone-induced colitis mice. Following the administration of modified Pulsatilla decoction or SASP, the elevated expression levels of colonic IL-5 and IL-13 were significantly attenuated. However, no significant differences were detected in the expression levels of colonic IL-5 and IL-13 between the modified Pulsatilla decoction and SASP-treated mice. Therefore, these results suggested that Pulsatilla decoction exerted anti-inflammatory effects in oxazolone-induced colitis.

Modified Pulsatilla decoction treatment restores the alteration of tight junction proteins in colon epithelial cells. Dysregulated gut epithelial permeability, which is determined by the intercellular tight junctions in chronic intestinal inflammation, has been suggested as a primary defect in IBD. To determine whether modified Pulsatilla decoction treatment can protect the integrity of the intestinal barrier, the present study examined the expression levels of the tight junction proteins, occludin, ZO-1 and claudin-2 in the colonic epithelial cells of mice treated with or without modified Pulsatilla decoction or SASP. As expected, the intercellular tight junctions were disrupted in the colon epithelium of the oxazolone-induced colitis mice, which was demonstrated by decreased expression levels of the tight junction proteins, occludin and ZO-1, and increased expression of claudin-2. However, treatment with modified Pulsatilla decoction or SASP restored the altered expression levels of occludin, ZO-1 and claudin-2 in the colon epithelial cells of the oxazolone-induced colitis mice, almost to normal levels (Fig. 3). These results indicated that modified Pulsatilla decoction prevented the alteration of tight junction proteins caused by the inflammatory stimuli in colon epithelial cells.

Modified Pulsatilla decoction treatment inhibits the activation of the $N F-\kappa B$ signaling pathway. The nuclear transcription factor, $\mathrm{NF}-\kappa \mathrm{B}$, is core in monitoring the development and progression of IBD by regulating the expression levels of a wide range of pro-inflammatory cytokines. $\mathrm{I} \kappa \mathrm{B} \alpha$ is one member of a family of cellular proteins, which function to inhibit the NF- $\kappa \mathrm{B}$ transcription factor. The stabilization of the $\mathrm{I} \kappa \mathrm{B}-\alpha$ subunit in the cytoplasm indicates the $\mathrm{NF}-\kappa \mathrm{B}$ signaling has been inhibited (25). For further insight into the molecular mechanism underlying the therapeutic effects of modified Pulsatilla decoction in IBD, the present study detected the expression of $\mathrm{I} \kappa \mathrm{B} \alpha$ in the colon tissues of mice treated with or 

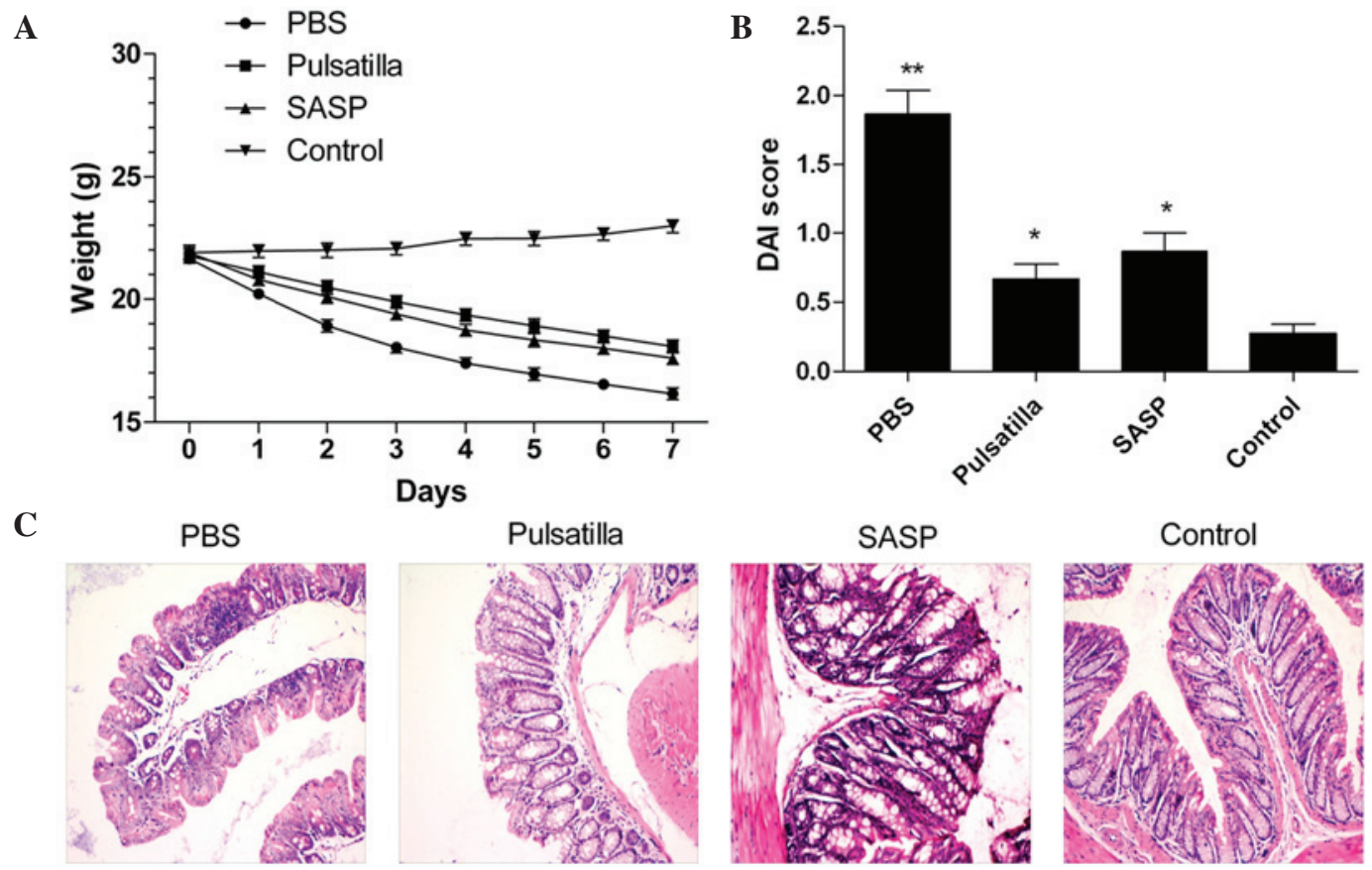

Figure 1. Modified Pulsatilla decoction treatment attenuates the severity of oxazolone-induced colitis in mice. (A) Weight loss of oxazolone-induced colitis mice following treatment with modified Pulsatilla decoction or SASP. (B) DAI scores were measured following 7 days of treatment with modified Pulsatilla decoction or SASP. (C) Representative images from the hematoxylin and eosin-stained sections of the colon from the different groups of mice. Magnification, $\mathrm{x} 200$. Data are representative of three independent experiments and expressed as the mean \pm standard deviation. ${ }^{*} \mathrm{P}<0.05{ }^{* *} \mathrm{P}<0.01$ vs. the control group. $\mathrm{PBS}$, phosphate-buffered saline; SASP, sulfasalazine; DAI, disease activity index.

without modified Pulsatilla decoction. As shown in Fig. 4, the expression of I $\mathrm{B}-\alpha$ was decreased in the colonic tissues of the oxazolone-induced colitis mice. However, stabilization of the I $\kappa \mathrm{B}-\alpha$ subunit in the colonic tissues of the modified Pulsatilla decoction- and SASP-treated mice was comparable to that of the normal mice. Therefore, modified Pulsatilla decoction inhibited the activation of the NF- $\mathrm{NB}$ signaling pathway, which contributed to its therapeutic effects on IBD.

\section{Discussion}

TCM is one of the most developed branches of CAM in managing various gastrointestinal disorders, and a wide range of natural products have shown effectiveness for the treatment of IBD (6-10). Modified Pulsatilla decoction is a TCM formulation based on the Pulsatilla decoction, with the addition of another three herbal plants (Sanchi, Radix Paeoniae Rubra and Radix Glycyrrhizae). It has been shown that the active ingredient from the herbal plants in the formulation of Pulsatilla decoction possesses a variety of pharmacological effects, including hepatic protective, anti-inflammatory, antibacterial, antitumor and anti-oxidant effects (15-19). Modified Pulsatilla decoction been used previously to treat UC in clinical practice in China, however, the possible underlying mechanism in the treatment of UC remains to be elucidated. The present study aimed to determine the efficiency and the underlying mechanisms of modified Pulsatilla decoction in experimental colitis.

Oxazolone-induced colitis is a hapten-induced murine model of colitis, with pathologic and immunologic features similar to UC (21). In the present study, colitis was induced in the mice by the intrarectal administration of a low dose of
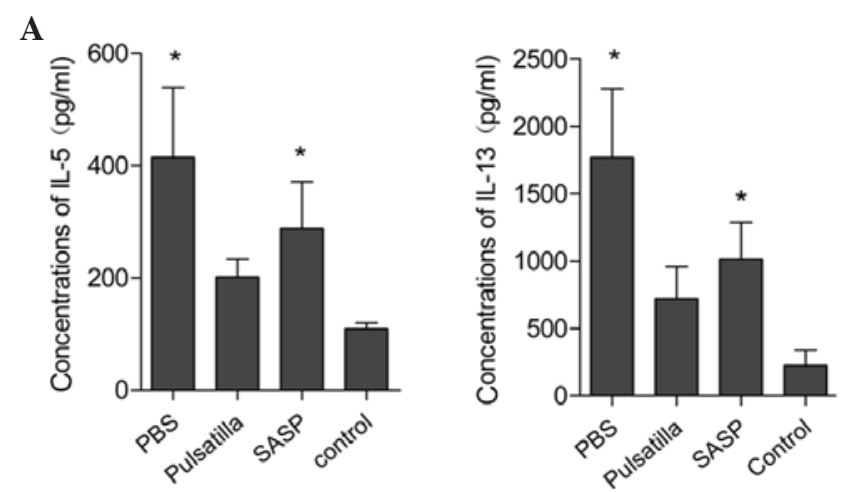

B
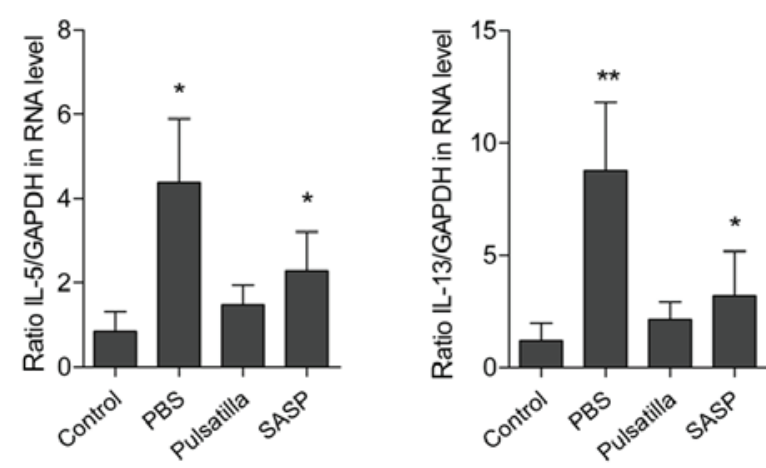

Figure 2. Modified Pulsatilla decoction treatment significantly reduces the secretion of pro-inflammatory cytokines. (A) mRNA expression levels of IL-5 and IL-13 in colon tissues from oxazolone-induced colitis mice, following treatment with or without modified Pulsatilla decoction or SASP, were determined using reverse transcription-quantitative polymerase chain reaction analysis. (B) Protein levels of IL-5 and IL-13 in colon tissues from oxazolone-induced colitis mice, following treatment with or without modified Pulsatilla decoction or SASP, were determined using ELISA. Data are expressed as the mean \pm standard deviation and are representative of three independent experiments. ${ }^{*} \mathrm{P}<0.05,{ }^{* *} \mathrm{P}<0.01$ vs. the control group. PBS, phosphate-buffered saline; SASP, sulfasalazine; IL, interleukin. 

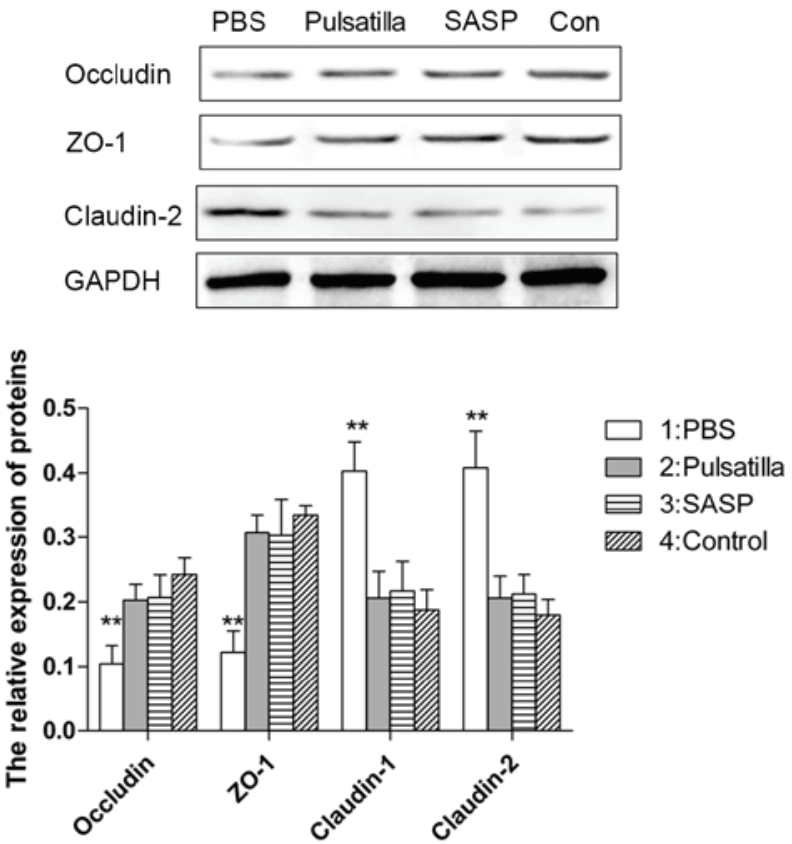

Figure 3. Modified Pulsatilla decoction treatment restores the alteration of tight junction proteins in colon epithelial cells. The expression levels of tight junction proteins, occludin, ZO-1 and claudin-2 in colonic epithelial cells of mice treated with or without modified Pulsatilla decoction or SASP were detected using western blot analysis. Data are expressed as the mean \pm standard deviation. ${ }^{* *} \mathrm{P}<0.01$ vs. the control group. PBS, phosphate-buffered saline; SASP, sulfasalazine; Con, control.

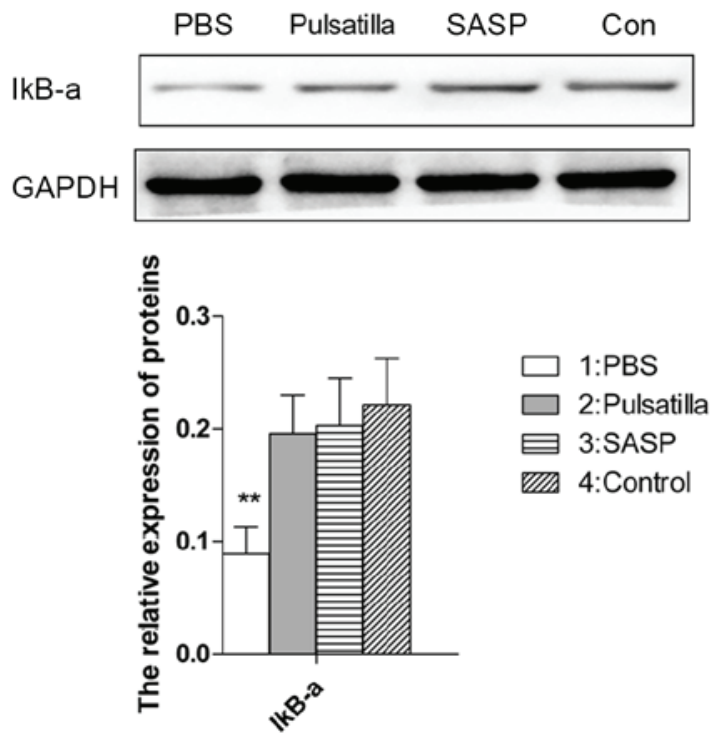

Figure 4. Modified Pulsatilla decoction treatment inhibits the activation of the nuclear factor- $\kappa \mathrm{B}$ signaling pathway. The expression of $\mathrm{I} \kappa \mathrm{B} \alpha$ in the colon tissues of oxazolone-induced colitis mice treated with or without modified Pulsatilla decoction were detected using western blot analysis. Data are expressed as the mean \pm standard deviation. ${ }^{* *} \mathrm{P}<0.01$ vs. the control group I $\mathrm{Ba}$, nuclear factor- $\kappa \mathrm{B} \alpha$; PBS, phosphate-buffered saline; SASP, sulfasalazine; Con, control.

oxazolone, following skin pre-sensitization with oxazolone. As expected, the colitis induced by oxazolone in the mice produced a significant loss in body weight, and the DAI score of the oxazolone-induced colitis mice was significantly higher, compared with that of the normal mice. Oxazolone-induced colitis has several similarities with human UC in terms of the morphological changes, which were characterized by transmural infiltrating of mononuclear cells, thickening of the colon wall, mucosal hyperplasia, erosions, depletion of goblet cells and crypt architectural distortion $(21,26)$. SASP is the first-line therapy for the induction and maintenance of remission in patients with UC $(27,28)$, however, conventional treatment of UC is currently limited to overcoming symptoms, and is often associated with severe adverse effects from the drugs used $(27,28)$. In previous years, the efficacy of Chinese herbal medicine in the treatment of IBD has been extensively characterized (6-10). The present study demonstrated the therapeutic effects of modified Pulsatilla decoction in treating oxazolone-induced colitis, which was demonstrated by improvements in symptomatic parameters, including body weight loss and severity of diarrhea, and histological damage of the colon. In addition, no differences between the therapeutic effects of SASP and modified Pulsatilla decoction were observed in treating oxazolone-induced colitis. Therefore, these findings indicated the efficiency of modified Pulsatilla decoction in treating experimental IBD.

An imbalance in T helper ( Th)1/Th2 cytokines is key in triggering the intestinal mucosa immune response, and $\mathrm{UC}$ is characterized by a Th2 immune response in which IL-13 and IL-5 have been identified as important cytokines (29-32). The Th2 cytokines, IL-13 and IL-5, are secreted at high levels by colonic lamina propria cells in patients with $\mathrm{UC}$, which cause epithelial cell cytotoxicity $(31,32)$. In UC, IL-13 may impair epithelial barrier function by affecting epithelial apoptosis, tight junctions and restitution velocity (32). Consistent with previous studies, the present study found that increased expression levels of IL-5 and IL-13 were observed in the colon tissues of the oxazolone-induced colitis mice. Following the administration of modified Pulsatilla decoction into the oxazolone-induced colitis mice, the elevated expression levels of colonic IL-5 and IL-13 were significantly attenuated, which suggested the anti-inflammatory effects of modified Pulsatilla decoction in treating IBD.

Intestinal inflammation is associated with defective intestinal epithelial tight junction barriers, which is an important pathogenic factor contributing to the development of IBD (33-35). The stable complex formed by occludin, ZO-1 and claudin-2 is critical for tight junction assembly, and the delocalization of occludin and ZO-1 from the tight junctions is associated with intestinal barrier dysfunction and increased permeability (33-35). Claudin-2 is a pore-forming protein, which enhances tight junction permeability for cations and provides the molecular basis for leak flux diarrhea in IBD $(36,37)$. Thus, upon stimulation by inflammatory cytokines, the structure of tight junctions are disrupted, which is demonstrated by decreased expression levels or internalization of occludin and ZO-1, and increased expression of the pore-forming, claudin-2. In the present study, the results showed that the tight junction proteins, occludin, ZO-1 and claudin-2, were significantly altered in the colon epithelial cells from the oxazolone-induced colitis mice, however, modified Pulsatilla decoction or SASP efficaciously prevented tight junction disruption.

The nuclear transcription factor, $\mathrm{NF}-\kappa \mathrm{B}$, is monitors the development and progression of IBD by regulating 
the expression of a wide range of pro-inflammatory cytokines $(38,39)$. In patients with IBD, increased NF- $\kappa$ B activation has been detected in the intestinal lamina propria $(38,39)$. It also has been shown that the increase of intestinal tight junction permeability is $\mathrm{NF}-\kappa \mathrm{B}-$ dependent $(40,41)$. In the present study, it was found that the $\mathrm{NF}-\kappa \mathrm{B}$ pathway was activated in the colon tissues from the oxazolone-induced colitis mice, and this activation was significantly counteracted by modified Pulsatilla decoction, indicating the possibility of this TCM formulation to downregulate the intestinal response to chronic inflammatory stimuli.

In conclusion, the present study determined the therapeutic efficiency of Pulsatilla decoction in oxazolone-induced colitis and examined the underlying mechanisms. In addition, no adverse effects were observed following treatment with modified Pulsatilla decoction. Although it is widely agreed the use of herbal medicines is safe for the treatment of various diseases in China, the long-term safety of modified Pulsatilla decoction requires further investigation.

\section{Acknowledgements}

This study was supported by a grant from Putuo Hospital (grant no. 2013PT054) and the ' 315 ' Program for Reserve Experts of Putuo Science \& Technology Commission (grant no. 14Q-RC-10).

\section{References}

1. Baumgart DC and Carding SR: Inflammatory bowel disease: Cause and immunobiology. Lancet 369: 1627-1640, 2007.

2. Xavier RJ and Podolsky DK: Unravelling the pathogenesis of inflammatory bowel disease. Nature 448: 427-434, 2007.

3. Baumgart DC and Sandborn WJ: Inflammatory bowel disease: Clinical aspects and established and evolving therapies. Lancet 369: 1641-1657, 2007.

4. Nielsen OH: New strategies for treatment of inflammatory bowel disease. Front Med (Lausanne) 1: 3, 2014.

5. Danese S: New therapies for inflammatory bowel disease: From the bench to the bedside. Gut 61: 918-932, 2012.

6. Hilsden RJ, Verhoef MJ, Rasmussen H, Porcino A and DeBruyn JC: Use of complementary and alternative medicine by patients with inflammatory bowel disease. Inflamm Bowel Dis 17: 655-662, 2011

7. Langmead L and Rampton DS: Review article: Complementary and alternative therapies for inflammatory bowel disease. Aliment Pharmacol Ther 23: 341-349, 2006.

8. Rahimi R, Mozaffari S and Abdollahi M: On the use of herbal medicines in management of inflammatory bowel diseases: A systematic review of animal and human studies. Dig Dis Sci 54: 471-480, 2009.

9. Jackson LN, Zhou Y, Qiu S, Wang Q and Evers BM: Alternative medicine products as a novel treatment strategy for inflammatory bowel disease. Am J Chin Med 36: 953-965, 2008.

10. Sałaga M, Zatorski H, Sobczak M, Chen C and Fichna J: Chinese herbal medicines in the treatment of IBD and colorectal cancer: A review. Curr Treat Options Oncol 15: 405-420, 2014.

11. Li R, Alex P, Ye M, Zhang T, Liu L and Li X: An old herbal medicine with a potentially new therapeutic application in inflammatory bowel disease. Int J Clin Exp Med 4: 309-319, 2011.

12. Cao YB, Zhang JD, Diao YY, Yan L, Wang DJ, Jia XM, Gao PH, Cheng MH, Xu Z, Wang Y and Jiang YY: Effects of Changtai granules, a traditional compound Chinese medicine, on chronic trinitrobenzene sulfonic acid-induced colitis in rats. World J Gastroenterol 11: 3539-3543, 2005.

13. Sandborn WJ, Targan SR, Byers VS, Rutty DA, Mu H, Zhang X and Tang T: Andrographis paniculata extract (HMPL-004) for active ulcerative colitis. Am J Gastroenterol 108: 90-98, 2013.
14. Zhang F, Li Y, Xu F, Chu Y and Zhao W: Comparison of Xilei-san, a Chinese herbal medicine and dexamethasone in mild/moderate ulcerative proctitis: A double-blind randomized clinical trial. J Altern Complement Med 19: 838-842, 2013.

15. Hu Y, Chen X, Duan H, Hu Y and Mu X: Pulsatilla decoction and its active ingredients inhibit secretion of NO, ET-1, TNF-alpha and IL-1 alpha in LPS-induced rat intestinal microvascular endothelial cells. Cell Biochem Funct 27: 284-288, 2009.

16. Cheng L, Zhang M, Zhang P, Song Z, Ma Z and Qu H: Silver complexation and tandem mass spectrometry for differentiation of triterpenoid saponins from the roots of Pulsatilla Chinensis (Bunge) Regel. Rapid Commun Mass Spectrom 22: 3783-3790, 2008.

17. Tan B, Ma Y, Shi R and Wang T: Simultaneous quantification of three alkaloids of Coptidis Rhizoma in rat urine by high-performance liquid chromatography: Application to pharmacokinetic study. Biopharm Drug Dispos 28: 511-516, 2007.

18. Chan CO, Chu CC, Mok DK and Chau FT: Analysis of berberine and total alkaloid content in cortex phellodendri by near infrared spectroscopy (NIRS) compared with high-performance liquid chromatography coupled with ultra-visible spectrometric detection. Anal Chim Acta 592: 121-131, 2007.

19. Shi Z, Zhu X and Zhang H: Micelle-mediated extraction and cloud point preconcentration for the analysis of aesculin and aesculetin in Cortex fraxini by HPLC. J Pharm Biomed Anal 44: 867-873, 2007.

20. Muluye RA, Bian Y and Alemu PN: Anti-inflammatory and antimicrobial effects of heat-clearing Chinese herbs: A current review. J Tradit Complement Med 4: 93-98, 2014.

21. Heller F, Fuss IJ, Nieuwenhuis EE, Blumberg RS and Strober W: Oxazolone colitis, a Th2 colitis model resembling ulcerative colitis, is mediated by IL-13-producing NK-T cells. Immunity 17: 629-638, 2002

22. National Research Council (US) Committee for the Update of the Guide for the Care and Use of Laboratory Animals. Guide for the care and use of laboratory animals. 8th edition. National Academies Press, Washington, D.C., USA, 2011.

23. Schmittgen TD and Livak KJ: Analyzing real-time PCR data by the comparative C(T) method. Nat Protoc 3: 1101-1108, 2008.

24. León AJ, Gómez E, Garrote JA, Bernardo D, Barrera A, Marcos JL, Férnandez-Salazar L, Velayos B, Blanco-Quirós A and Arranz E: High levels of proinflammatory cytokines, but not markers of tissue injury, in unaffected intestinal areas from patients with IBD. Mediators Inflamm 2009: 30, 2009.

25. Imbert V, Rupec RA, Livolsi A, Pahl HL, Traenckner EB, Mueller-Dieckmann C, Farahifar D, Rossi B, Auberger P, Baeuerle PA and Peyron JF: Tyrosine phosphorylation of I kappa B-alpha activates NF-kappa B without proteolytic degradation of I kappa B-alpha. Cell 86: 787-798, 1996.

26. Kojima R, Kuroda S, Nakamaru $K$ and Hatakeyama S: Oxazolone-induced colitis in BALB/C mice: A new method to evaluate the efficacy of therapeutic agents for ulcerative colitis. J Pharmacol Sci 96: 307-313, 2004.

27. Svartz N: Salazopyrin a new sulfanilamide preparation: A. Therapeutic results in rheumatic polyarthritis. B. Therapeutic results in ulcerative colitis. C. Toxic manifestations in treatment with sulfanilamide preparation. Acta Med Scand 11: 557-590, 1942.

28. Kornbluth A and Sachar DB; Practice Parameters Committee of the American College of Gastroenterology: Ulcerative colitis practice guidelines in adults: American college of gastroenterology, practice parameters committee. Am J Gastroenterol 105: 501-523; quiz 524, 2010.

29. Khor B, Gardet A and Xavier RJ: Genetics and pathogenesis of inflammatory bowel disease. Nature 474: 307-317, 2011.

30. Strober W and James SP: The immunologic basis of inflammatory bowel disease. J Clin Immunol 6: 415-432, 1986.

31. Fuss IJ, Neurath M, Boirivant M, Klein JS, de la Motte C, Strong SA, Fiocchi C and Strober W: Disparate CD4+ lamina propria (LP) lymphokine secretion profiles in inflammatory bowel disease. Crohn's disease LP cells manifest increased secretion of IFN-gamma, whereas ulcerative colitis LP cells manifest increased secretion of IL-5. J Immunol 157: 1261-1270, 1996.

32. Heller F, Florian P, Bojarski C, Richter J, Christ M, Hillenbrand B, Mankertz J, Gitter AH, Burgel N, Fromm M, et al: Interleukin-13 is the key effector Th2 cytokine in ulcerative colitis that affects epithelial tight junctions, apoptosis and cell restitution. Gastroenterology 129: 550-564, 2005. 
33. Hering NA, Fromm M and Schulzke JD: Determinants of colonic barrier function in inflammatory bowel disease and potential therapeutics. J Physiol 590: 1035-1044, 2012.

34. Suzuki T: Regulation of intestinal epithelial permeability by tight junctions. Cell Mol Life Sci 70: 631-659, 2013

35. Turner JR: Intestinal mucosal barrier function in health and disease. Nat Rev Immunol 9: 799-809, 2009.

36. Weber CR, Raleigh DR, Su L, Shen L, Sullivan EA, Wang Y and Turner JR: Epithelial myosin light chain kinase activation induces mucosal interleukin-13 expression to alter tight junction ion selectivity. J Biol Chem 285: 12037-12046, 2010.

37. Prasad S, Mingrino R, Kaukinen K, Hayes KL, Powell RM, MacDonald TT and Collins JE: Inflammatory processes have differential effects on claudins 2, 3 and 4 in colonic epithelial cells. Lab Invest 85: 1139-1162, 2005.
38. Schreiber S, Nikolaus S and Hampe J: Activation of nuclear factor kappa B inflammatory bowel disease. Gut 42: 477-484, 1998.

39. Neurath MF, Becker C and Barbulescu K: Role of NF-kappaB in immune and inflammatory responses in the gut. Gut 43: 856-860, 1998.

40. Ma TY, Iwamoto GK, Hoa NT, Akotia V, Pedram A, Boivin MA and Said HM: TNF-alpha-induced-induced increase in intestinal epithelial tight junction permeability requires NF-kappa B activation. Am J Physiol Gastrointest Liver Physiol 286: G367-G376, 2004.

41. Beutheu Youmba S, Belmonte L, Galas L, Boukhettala N, Bôle-Feysot C, Déchelotte P and Coëffier M: Methotrexate modulates tight junctions through NF- $\kappa \mathrm{B}, \mathrm{MEK}$, and JNK Pathways. J Pediatr Gastroenterol Nutr 54: 463-470, 2012. 